\title{
Hypertension in patients with CKD in China: clinical characteristics and management
}

\author{
Guangyan Cai $(\bowtie)^{\mathrm{a}}$, Xiangmei Chen $(\bowtie)^{\mathrm{b}}$ \\ Department of Nephrology, Chinese PLA General Hospital, Chinese PLA Institute of Nephrology, State Key Laboratory of Kidney Diseases, \\ National Clinical Research Center for Kidney Diseases, Beijing 100853, China \\ (C) Higher Education Press and Springer-Verlag Berlin Heidelberg 2017
}

Chronic kidney disease (CKD) is a life-threatening disease that results in end-stage renal disease (ESRD) and consumes substantial health resources. In China, the prevalence of CKD in the general population is $10.8 \%$ and increases gradually with age [1]. CKD also causes hypertension. The prevalence of hypertension in the general population is $25.2 \%$ [2], and the prevalence of hypertension with CKD is much higher. From 2010 to 2011, a national epidemiological survey of CKD in hospitalized patients (PATRIOTIC study) showed that the prevalence of renal hypertension was $67.3 \%$ [3]. Another study from 2012 to 2013 indicated that the prevalence of hypertension in CKD patients was 71.2\% [4], suggesting the elevating prevalence of renal hypertension.

The PATRIOTIC study was a multi-center epidemiological survey, which included 61 hospitals in China. The results showed that the prevalence of renal hypertension in the elderly was $82.0 \%$, significantly higher than that of young patients with CKD. The prevalence rates of hypertension in the elderly CKD groups aged 60-69 years, 70-79 years, and over 80 years old were $81.2 \%$, $82.6 \%$, and $84.5 \%$, respectively, indicating that the older the age, the higher the prevalence rate. As eGFR falls, the prevalence rate of hypertension increases [5]. Compared with those in CKD stage 1, the prevalence rate of patients in CKD stages 3b, 4, and 5 rises significantly [6].

Although the prevalence of renal hypertension remains high, awareness and treatment rate have been improved in recent years. The awareness and treatment rate of renal hypertension in the survey from 1999 to 2002 were $75.7 \%$ and $80.4 \%$ [7], respectively, which rose to $95.4 \%$ and $93.7 \%$ in the survey from 2012 to 2013 [4]. These findings suggested the dramatic improvement of the condition. Nevertheless, a survey from hospitalized patients with

Correspondence: acaiguangyan@sina.com; bxmchen301@126.com
CKD showed that $4.6 \%$ had no knowledge of hypertension. The awareness of renal hypertension in the community was likely much lower. A telephone survey conducted in Hong Kong for the general population revealed that $43.6 \%$ of the subjects did not understand the importance of blood pressure measurement in CKD, and more than $60 \%$ were unaware that hypertension is a complication of CKD [8]. This finding indicates that efforts on the publicity, prevention, and control of hypertension in the community need to be further improved.

Improving the management of hypertension plays an important role in delaying renal disease progression and complications, as well as enhances the prognosis of CKD patients. To promote hypertension management, a number of guidelines for prevention and treatment were developed, among which the Kidney Disease: Improving Global Outcomes (KDIGO) Guideline (2012) and the United States NKF KDOQI (2004) were the most influential $[9,10]$. The Chinese Nephrologist Association initiated and completed the Chinese Guidelines for the Management of Hypertension in CKD (2016), hoping to guide clinical practice and improve awareness, diagnosis, and treatment of hypertension in CKD in China. The KDIGO guidelines recommend that for non-dialysis CKD patients with hypertension and normal or mild albuminuria, regardless of diabetes mellitus, blood pressure control target should be less than 140/90 mmHg. For those with moderate to severe proteinuria, antihypertensive treatment target should be less than 130/80 $\mathrm{mmHg}$. The Chinese Guidelines for the Management of Hypertension in CKD also recommend a blood pressure control target $<140 / 90$ $\mathrm{mmHg}$ in CKD patients. When the urinary albumin excretion rate $>300 \mathrm{mg} / 24 \mathrm{~h}$, the blood pressure control target is below $130 / 80 \mathrm{mmHg}$.

The control rate of hypertension in CKD in China is continually improved. The 1999-2000 survey showed that the control rates for $140 / 90$ and $130 / 80 \mathrm{mmHg}$ targets were 
$21.1 \%$ and $5.9 \%$, respectively [7]. The control rates were increased to $41.1 \%$ and $15 \%$ in the 2012-2013 survey [4]. However, the control rate of hypertension in Chinese CKD patients was still lower than in developed countries. Taking the CRIC study in the US as an example, the control rates for $140 / 90$ and $130 / 80 \mathrm{mmHg}$ treatment targets were $67.1 \%$ and $46.1 \%$, respectively. Many factors affect the control of hypertension in CKD. Iatrogenic factors include inappropriate prescription of antihypertensive drugs, a low proportion of combined treatment, and inadequate use of diuretics. Patient-related factor is mainly poor compliance for medication. Hypertension in CKD is always associated with refractory factors, such as fluid overload, increased vasoconstrictor material, and reduced vasodilator material. With regard to antihypertensive treatment for hypertension in $\mathrm{CKD}$, combination treatment is commonly used for optimal blood pressure control. Nevertheless, a recent study showed that the ratios of monotherapy and two-, three-, four-, five-, and six-drug combination treatments were $37.7 \%, 38.7 \%, 15.8 \%, 3.9 \%, 0.7 \%$, and $0.1 \%$ [4], respectively, indicating the dominance of mono- and twodrug combinations in hypertension control in Chinese CKD patients. The use of three or more drug combinations are seldom used. The mechanism of hypertension in CKD is associated with the activated RAS system and fluid capacity load. Reasonable combination of antihypertensive therapy should be preferred, which will make better control of blood pressure in CKD.

The incidence of nocturnal hypertension and abnormal changes of blood pressure rhythm is high in CKD patients in China. The prevalence of isolated nocturnal hypertension in CKD patients was found to be closely related to target organ damage. For example, Wang et al. reported the prevalence rate of 723 CKD patients with solitary night hypertension was $20.44 \%$ [5]. Time therapy is a better solution for nocturnal hypertension than non-spoon-type blood pressure control. A study on 60 subjects with nonspoon-type blood pressure in CKD showed that although valsartan significantly reduced systolic and diastolic blood pressure before and after bedtime administration, it significantly improved non-spoon-type blood pressure [11]. A meta-analysis also confirmed that time therapy significantly reduced nocturnal systolic and diastolic blood pressure [12]. Nocturnal hypertension and non-spoon-type blood pressure control is improved by this treatment method.

Another reason for the low control rate of hypertension in Chinese CKD patients is the low usage of diuretics. Compared with the CRIC study, the use of diuretics was not enough in CKD patients involved in the PATRIOTIC study. Uncontrolled hypertension in CKD patients is characterized by water and sodium retention and increased blood volume. A study of patients who underwent hemodialysis treatment revealed that blood pressure was further reduced in low dialysis sodium concentrations and even in patients who have reached their dry weight [13]. Therefore, the use of diuretics is considered reasonable in controlling CKD hypertension.

In addition, the viewpoints of doctors and patients affect the control of blood pressure. The HAPPEN study disclosed that the expectation for blood pressure control rate differs significantly between doctors and patients. In this investigation, the doctors claimed to have informed the blood pressure target goal in $90 \%$ of patients; however, only $67 \%$ of patients knew their target goals, among which only $31 \%$ reached the target in the first two weeks of measurement [14]. Moreover, some patients knew that they suffered from hypertension but still refused to take treatment. Educating doctors and patients in managing and controlling hypertension in CKD is very important.

Poor control of blood pressure is related to bad prognosis of patients with $\mathrm{CKD}$, leading to kidney disease progression and, eventually, ESRD and death [15-17]. Cardiovascular and cerebrovascular complications and ESRD can be reduced by better blood pressure control in patients with CKD. The application of ACEI/ARB and other antihypertensive drugs, appropriate use of diuretics, and limitation of sodium intake lay the basis for hypertension treatment in CKD. Combination therapy facilitates blood pressure control in advanced stages of CKD. Administration of antihypertensive drugs before bedtime allows patients to control their blood pressure. In a word, the prevention and treatment of hypertension in CKD need collaboration and concerted effort between doctors and patients.

\section{Acknowledgements}

This work was supported by the National Science and Technology Support Program (Nos. 2015BAI12B06 and 2013BAI09B05), the National Natural Science Foundation of China (No. 81330019), the Science and Technology Project of Beijing (No. D131100004713003), and the 973 program (No. 2013CB530800).

\section{Compliance with ethics guidelines}

Guangyan Cai and Xiangmei Chen declare that they have no conflicts of interest. This manuscript is an editorial and does not involve a research protocol requiring approval by the relevant institutional review board or ethics committee.

\section{References}

1. Zhang L, Wang F, Wang L, Wang W, Liu B, Liu J, Chen M, He Q, Liao Y, Yu X, Chen N, Zhang JE, Hu Z, Liu F, Hong D, Ma L, Liu H, Zhou X, Chen J, Pan L, Chen W, Wang W, Li X, Wang H. Prevalence of chronic kidney disease in China: a cross-sectional survey. Lancet 2012; 379(9818): 815-822

2. Wang J, Zhang L, Wang F, Liu L, Wang H; the China National 
Survey of Chronic Kidney Disease Working Group. Prevalence, awareness, treatment, and control of hypertension in China: results from a national survey. Am J Hypertens 2014; 27(11): 1355-1361

3. Zheng Y, Cai GY, Chen XM, Fu P, Chen JH, Ding XQ, Yu XQ, Lin HL, Liu J, Xie RJ, Wang LN, Ni ZH, Liu FY, Yin AP, Xing CY, Wang L, Shi W, Liu JS, He YN, Ding GH, Li WG, Wu GL, Miao LN, Chen N, Su Z, Mei CL, Zhao JY, Gu Y, Bai YK, Luo HM, Lin S, Chen MH, Gong L, Yang YB, Yang XP, Li Y, Wan JX, Wang NS, Li HY, Xi CS, Hao L, Xu Y, Fang JA, Liu BC, Li RS, Wang R, Zhang JH, Wang JQ, Lou TQ, Shao FM, Mei F, Liu ZH, Yuan WJ, Sun SR, Zhang L, Zhou CH, Chen QK, Jia SL, Gong ZF, Guan GJ, Xia T, Zhong LB; Prevalence, Awareness, and Treatment Rates in Chronic Kidney Disease Patients with Hypertension in China (PATRIOTIC) Collaborative Group. Prevalence, awareness, treatment, and control of hypertension in the non-dialysis chronic kidney disease patients. Chin Med J (Engl) 2013; 126(12): 2276-2280

4. Zhang W, Shi W, Liu Z, Gu Y, Chen Q, Yuan W, Zhang Y, Gong L, Zhou R, Li M, Cheng H, Liu J, Cen J, Huang C, Ren Y, Mao P, Xing C, Hong F, Jiang D, Wang L, Xu G, Liu J, Chen N. A nationwide cross-sectional survey on prevalence, management and pharmacoepidemiology patterns on hypertension in Chinese patients with chronic kidney disease. Sci Rep 2016; 6(1): 38768

5. Wang C, Deng WJ, Gong WY, Zhang J, Tang H, Peng H, Zhang QZ, Ye ZC, Lou T. High prevalence of isolated nocturnal hypertension in Chinese patients with chronic kidney disease. J Am Heart Assoc 2015; 4(6): e002025

6. Cai G, Zheng Y, Sun X, Chen X; Survey of Prevalence, Awareness, and Treatment Rates in Chronic Kidney Disease Patients with Hypertension in China Collaborative Group. Prevalence, awareness, treatment, and control of hypertension in elderly adults with chronic kidney disease: results from the survey of Prevalence, Awareness, and Treatment Rates in Chronic Kidney Disease Patients with Hypertension in China. J Am Geriatr Soc 2013; 61(12): 2160-2167

7. Wang Y, Zhang L, Li X, Xu Y, Yang M, Qian J, Wang L, Chen N, Gu Y, Chen M, Xing C, Chen X, Hou F, Yu X, Cheng X, Guo L, Wei C, Huang G, Zhang Q, Wang R, Wang L, Mei C, Li Y, Liu Z, Zhao $\mathrm{L}$, Wu Y, Wang HY. Improvement of awareness, treatment and control of hypertension among chronic kidney disease patients in China from 1999 to 2005. Hypertens Res 2009; 32(6): 444-449

8. Chow KM, Szeto CC, Kwan B, Leung CB, Li PK. Public lacks knowledge on chronic kidney disease: telephone survey. Hong Kong Med J 2014; 20(2): 139-144
9. Kidney Disease: Improving Global Outcomes (KDIGO) Blood Pressure Work Group. KDIGO Clinical Practice Guideline for the Management of Blood Pressure in Chronic Kidney Disease. Kidney Int Suppl 2012;2:337-414

10. Kidney Disease Outcomes Quality Initiative (K/DOQI). K/DOQI clinical practice guidelines on hypertension and antihypertensive agents in chronic kidney disease. Am J Kidney Dis 2004; $43(5$ Suppl 1): S1-S290

11. Wang C, Zhang J, Liu X, Li CC, Ye ZC, Peng H, Chen Z, Lou T. Effect of valsartan with bedtime dosing on chronic kidney disease patients with nondipping blood pressure pattern. J Clin Hypertens (Greenwich) 2013; 15(1): 48-54

12. Wang C, Qiu X, Lv L, Huang J, Li S, Lou T, Liu X. Chronotherapy for hypertension in patients with chronic kidney disease: a systematic review and meta-analysis in non-black patients. Int Urol Nephrol 2017; 49(4): 651-659

13. Zhou YL, Liu J, Ma LJ, Sun F, Shen Y, Huang J, Cui TG. Effects of increasing diffusive sodium removal on blood pressure control in hemodialysis patients with optimal dry weight. Blood Purif 2013; 35 (1-3): 209-215

14. Zhang X, Feldmam R, Wu Z, Zhang Y, Yu X, Liu L. Therapeutic inertia is a major determinant if $\mathrm{BP}$ control rates in China. A hypertension attitude perspectives and needs (HAPPEN) study report. J Hypertens 2015; 33(Suppl 1):e128

15. Drawz PE, Alper AB, Anderson AH, Brecklin CS, Charleston J, Chen J, Deo R, Fischer MJ, He J, Hsu CY, Huan Y, Keane MG, Kusek JW, Makos GK, Miller ER 3rd, Soliman EZ, Steigerwalt SP, Taliercio JJ, Townsend RR, Weir MR, Wright JT Jr, Xie D, Rahman M; Chronic Renal Insufficiency Cohort Study Investigators. Masked hypertension and elevated nighttime blood pressure in CKD: prevalence and association with target organ damage. Clin J Am Soc Nephrol 2016; 11(4): 642-652

16. Wang C, Li Y, Zhang J, Ye ZC, Zhang QZ, Ma XX, Peng H, Lou TQ. Prognostic effect of isolated nocturnal hypertension in Chinese patients with nondialysis chronic kidney disease. J Am Heart Assoc 2016; 5(10): e004198

17. Minutolo R, Gabbai FB, Agarwal R, Chiodini P, Borrelli S, Bellizzi V, Nappi F, Stanzione G, Conte G, De Nicola L. Assessment of achieved clinic and ambulatory blood pressure recordings and outcomes during treatment in hypertensive patients with CKD: a multicenter prospective cohort study. Am J Kidney Dis 2014; 64(5): $744-752$ 